\title{
Variations in macadamia varietal susceptibility to Phytophthora multivora and P. cinnamomi
}

\author{
Olumide Jeff-Ego ${ }^{1, *}$, Juliane Henderson ${ }^{2}$, Bruce Topp ${ }^{1}$, Andre Drenth ${ }^{1}$ and Olufemi Akinsanmi ${ }^{1}$ \\ 1 Centre for Horticultural Science, Queensland Alliance for Agriculture \& Food Innovation, Queensland \\ Alliance for Agriculture \& Food Innovation, The University of Queensland, Dutton Park, QLD 4072, \\ Australia; b.topp@uq.edu.au (B.T.); a.drenth@uq.edu.au (A.D.); o.akinsanmi@uq.edu.au (O.A.) \\ 2 Department of Agriculture and Fisheries, Dutton Park, QLD 4072, Australia; \\ Juliane.Henderson@daf.qld.gov.au \\ * Correspondence: o.jeffego@uq.net.au \\ + Presented at the third International Tropical Agriculture Conference (TROPAG 2019), Brisbane, Australia, \\ 11-13 November 2019.
}

Published: 21 February 2020

\begin{abstract}
Incidence of diseases caused by various Phytophthora spp. in macadamia is increasing worldwide, often resulting in severe yield loss and death of both juvenile and old macadamia trees. In Australia, P. cinnamomi and P. multivora has been identified to cause severe stem canker in macadamia orchards. Currently, various varietal improvement research programs on macadamia are underway, not only to obtain high-yielding and precocious macadamia germplasm but to develop macadamia genotypes with acceptable levels of tolerance/resistance to pests and diseases. In this study, we selected macadamia genotypes for their acceptable levels of tolerance/resistance to Phytophthora infection, after screening about 360 macadamia genotypes against to $P$. cinnamomi and $P$. multivora, using a rapid detached leaf and plant inoculation assays. These macadamia plant materials were collected from a major macadamia arboretum in Queensland. Our results revealed segregation of macadamia genotypes into two spectrum of susceptible and tolerant macadamia genotypes. Hence, strong representatives within the tolerant and susceptible spectrum were further investigated in order to understand if the basis of the differential response is driven by a variation in structural, physiological or genetic markers, following a challenge with Phytophthora spp. Our result revealed a marked variation in structural response during the infection process, between the selected susceptible, 'HAES816' and tolerant germplasm and 'HAES344'. This information will provide evidence to support potential preferential selection of tolerant macadamia genotypes potentially useful for breeding against Phytophthora diseases. Further studies will characterize tolerant macadamia progenies to develop marker-assisted selection protocols for resistance, and explore defense mechanisms response.
\end{abstract}

Keywords: proteacea; pathogen; tree nuts

Funding: This work was supported by grant from the Hort Innovation using the Macadamia levy Fund, and Commonwealth government for Project MC 16018.

Acknowledgments: UQ Research Training Scholarship for O.J.E., and the Department of Primary Industries, New South Wales, Australia are gratefully acknowledged.

Conflicts of Interest: The author declares no conflict of interest.

(C) 2019 by the authors. Licensee MDPI, Basel, Switzerland. This article is an open access article distributed under the terms and conditions of the Creative Commons Attribution (CC BY) license (http://creativecommons.org/licenses/by/4.0/). 